\title{
Editorial
}

\section{Peptide-Based Immunotherapeutics and Vaccines}

\author{
Pedro A. Reche, ${ }^{1}$ Enrique Fernandez-Caldas, ${ }^{2}$ Darren R. Flower, ${ }^{3}$ \\ Masha Fridkis-Hareli, ${ }^{4}$ and Yoshihiko Hoshino ${ }^{5}$ \\ ${ }^{1}$ Departamento de Microbiología I, Inmunología, Facultad de Medicina, Universidad Complutense, 28040 Madrid, Spain \\ ${ }^{2}$ Inmunotek SL, Alcalá de Henares, 28805 Madrid, Spain \\ ${ }^{3}$ School of Life and Health Sciences, Aston University, Birmingham B4 7ET, UK \\ ${ }^{4}$ ATR, LLC, Worcester, MA 01606, USA \\ ${ }^{5}$ Department of Mycobacteriology, National Institute of Infectious Diseases, Higashi-Murayama, Tokyo 189-0002, Japan
}

Correspondence should be addressed to Pedro A. Reche; parecheg@med.ucm.es

Received 16 December 2013; Accepted 16 December 2013; Published 4 February 2014

Copyright (c) 2014 Pedro A. Reche et al. This is an open access article distributed under the Creative Commons Attribution License, which permits unrestricted use, distribution, and reproduction in any medium, provided the original work is properly cited.

The discovery of small molecule and biologic drugs offers treatments applicable to a wide variety of infectious and chronic human diseases. Antibiotics have, since World War II, been the weapons of choice in humanity's on-going battle with pathogenic bacteria, and more recently the discovery of antiviral drugs has been an area of active endeavor. Yet the emergence of multiresistant bacteria, as well as the continuing prevalence of antigenic drift in viruses, has shown the fundamental limitations of postinfection drug therapy. Fortunately, the scientific exploitation of host immunity provides an even more powerful approach to forestalling infection and counteracting chronic disease states. The supreme embodiment of this is the vaccine. Indeed, prophylactic, and to a lesser extent therapeutic, vaccines continue to be the most cost-effective and efficient alternative to other medicines for the treatment and prevention of infectious diseases, cancer, and allergy conditions. Importantly, unlike drug-based medicines, vaccines are primarily curative not palliative remedies.

Vaccines work by inducing profound changes in the cellular components of adaptive immunity, comprising $\mathrm{T}$ - and B-cells. The first vaccines targeted infections pathogens and were composed of killed or attenuated pathogens. However, our emerging understanding of antigen presentation and subsequent recognition has enabled the development of several new types of vaccine, including those based on single proteins or on synthetic peptides encompassing many B- and T-cell epitopes [1]. Peptide-based epitope ensemble vaccines offer several potential advantages over traditional whole-organism vaccines. Chiefly, they allow the immune response to focus solely on relevant epitopes, avoiding those that lead to nonprotective responses, immune evasion, or unwanted side effects, such as autoimmunity. Consequently, peptide-based vaccines in particular offer renewed hope for the treatment and prevention of chronic infectious diseases such as those caused by hepatitis $C$ virus and cancer [2, 3]. Likewise, peptide-based vaccines can also be used as safer ways of inducing allergen specific tolerance [3].

The design of peptide-based vaccines takes advantage of an emergent computational paradigm that couples immunoinformatic prediction with rigorous experimental validation, thus facilitating the identification of epitopes within protein antigens [1]. Amongst extant prediction technologies, the most fruitful has been data-driven prediction of T-cell epitopes. T-cell epitope prediction relies primarily on the anticipation of peptide-binding to MHC molecules [4] and many methods drawn from bioinformatics and/or chemoinformatics are known to produce satisfactory results $[5,6]$.

However, development of effective peptide-based vaccines requires science to overcome a number of significant difficulties, including identifying optimal delivery routes, overcoming the low intrinsic immunogenicity of individual peptides, and the task of combining different types of epitopes to engage the humoral and cellular arms of the adaptive immune response properly, as well as compensating for the poor population coverage of individual T-cell epitopes due to $\mathrm{MHC}$ restriction. On a more technical level, certain antigenic 
epitopes, specifically B-cell epitopes or antibody determinants, are conformational and need to be mimicked by linear sequences or placed onto suitable spatial frameworks. Moreover, methods for predicting B-cell epitopes need to be improved as they are notoriously unreliable in predicting both linear [7] and conformational [8,9] B-cell epitopes.

In this special issue on peptide-based vaccines, we have gathered together a varied array of cutting-edge reports and reviews detailing several important and innovative approaches to the design and discovery of epitope-based vaccines. Articles range from reports on new computational methods for epitope prediction and selection for vaccine design to clinical trials using peptide-based vaccines.

We hope that readers will find this special issue not only interesting but also enlightening, exciting, and inspiring. As more and more pathogen genomes are revealed and our understanding of fundamental immunological mechanisms deepens, it becomes ever clearer that the only tractable approach to vaccine discovery and design is through the interplay of in silico with in vitro and in vivo techniques. Indeed, the combination of computational and experimental epitope mapping offers many tools and techniques appropriate to the identification and optimization of vaccine candidates. As this special issue demonstrates so well, such methodology is fast becoming one of the most pivotal tools currently available to vaccine developers; and it foreshadows the day when this variety of approaches will fully synergize to speed up the development of powerfully efficacious vaccines against both infective and chronic disease states.

\section{Pedro A. Reche Enrique Fernandez-Caldas Darren R. Flower Masha Fridkis-Hareli Yoshihiko Hoshino}

\section{References}

[1] D. R. Flower, "Designing immunogenic peptides," Nature Chemical Biology, vol. 9, no. 12, pp. 749-753, 2013.

[2] C. Y. Wang and A. M. Walfield, "Site-specific peptide vaccines for immunotherapy and immunization against chronic diseases, cancer, infectious diseases, and for veterinary applications," Vaccine, vol. 23, no. 17-18, pp. 2049-2056, 2005.

[3] Y. Hailemichael and W. W. Overwijk, "Peptide-based anticancer vaccines: the making and unmaking of a T-cell graveyard," Oncoimmunology, vol. 2, no. 7, Article ID e24743, 2013.

[4] E. M. Lafuente and P. A. Reche, "Prediction of MHC-peptide binding: a systematic and comprehensive overview," Current Pharmaceutical Design, vol. 15, no. 28, pp. 3209-3220, 2009.

[5] B. Peters, H.-H. Bui, S. Frankild et al., "A community resource benchmarking predictions of peptide binding to MHC-I molecules," PLoS Computational Biology, vol. 2, no. 6, article e65, 2006.

[6] H. H. Lin, G. L. Zhang, S. Tongchusak, E. L. Reinherz, and V. Brusic, "Evaluation of MHC-II peptide binding prediction servers: applications for vaccine research," BMC Bioinformatics, vol. 9, article S22, 2008.
[7] M. J. Blythe and D. R. Flower, "Benchmarking B cell epitope prediction: underperformance of existing methods," Protein Science, vol. 14, no. 1, pp. 246-248, 2005.

[8] J. V. Ponomarenko and P. E. Bourne, "Antibody-protein interactions: benchmark datasets and prediction tools evaluation," BMC Structural Biology, vol. 7, article 64, 2007.

[9] P. Sun, H. Ju, Z. Liu et al., "Bioinformatics resources and tools for conformational B-cell epitope prediction," Computational and Mathematical Methods in Medicine, vol. 2013, Article ID 943636, 11 pages, 2013. 


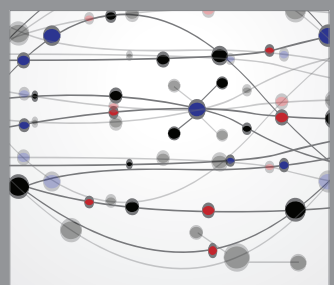

The Scientific World Journal
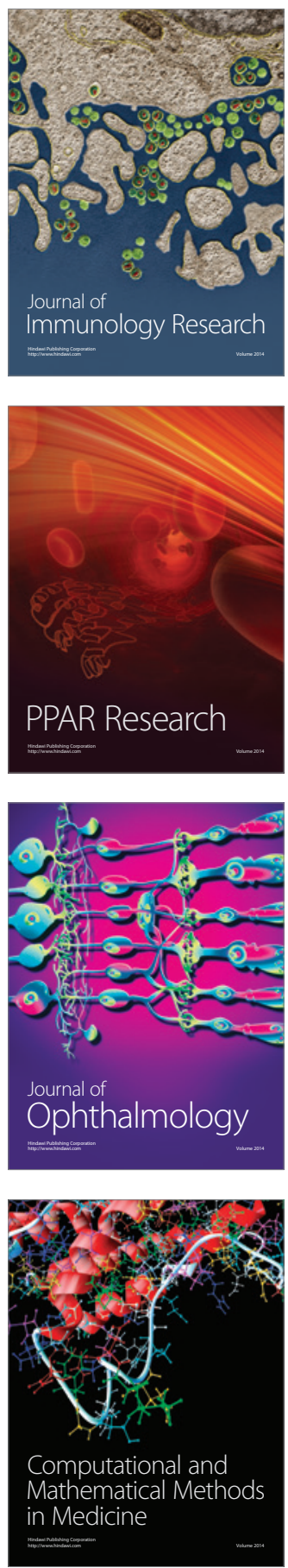

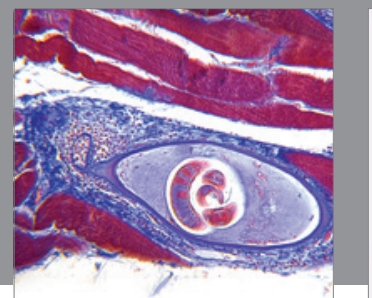

Gastroenterology

Research and Practice
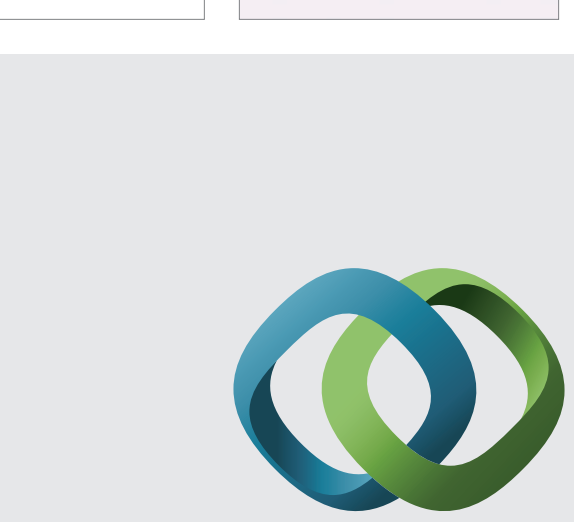

\section{Hindawi}

Submit your manuscripts at

http://www.hindawi.com
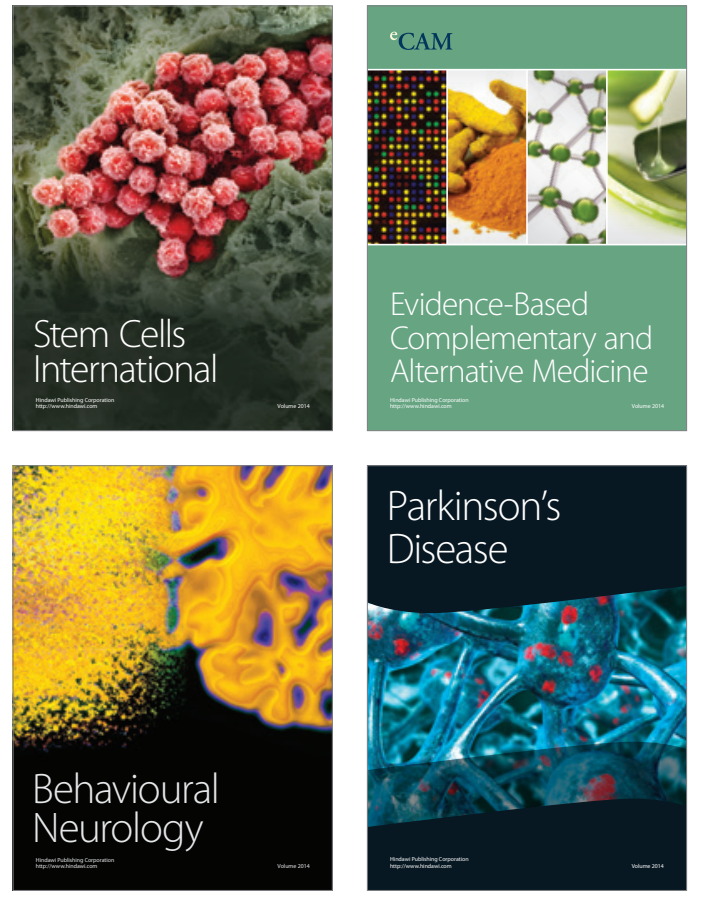
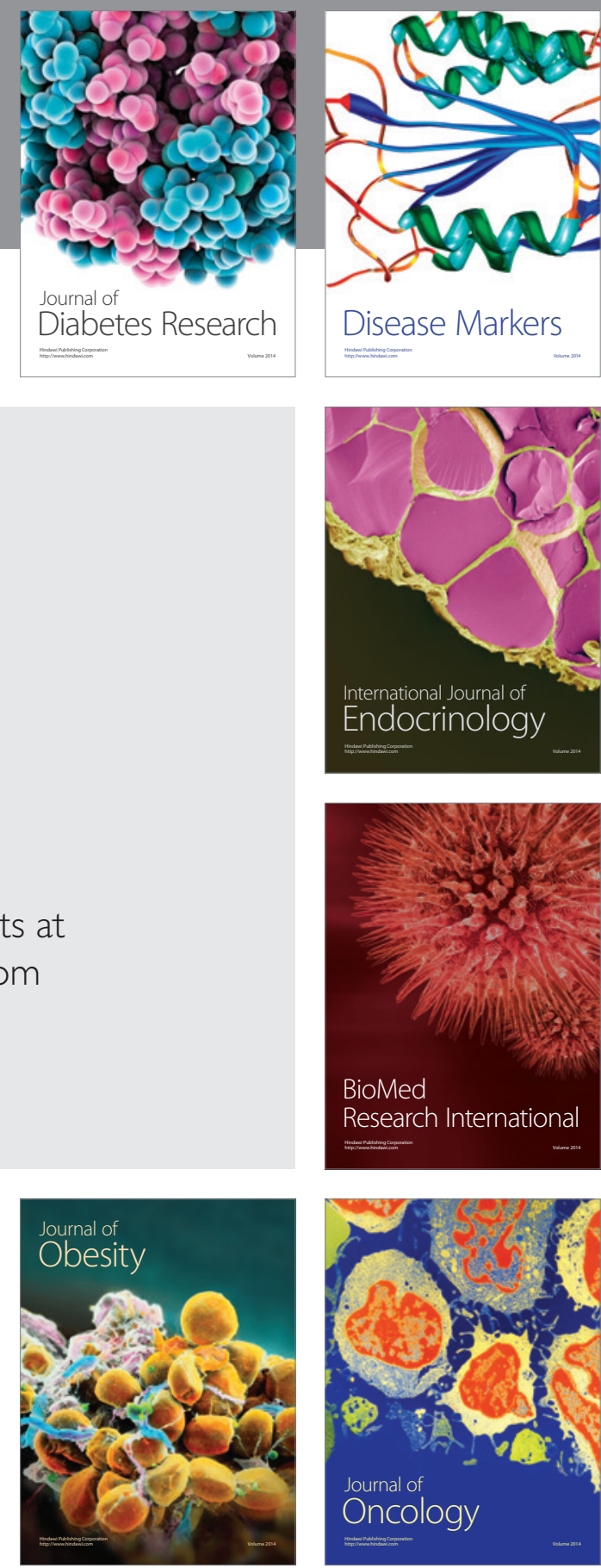

Disease Markers
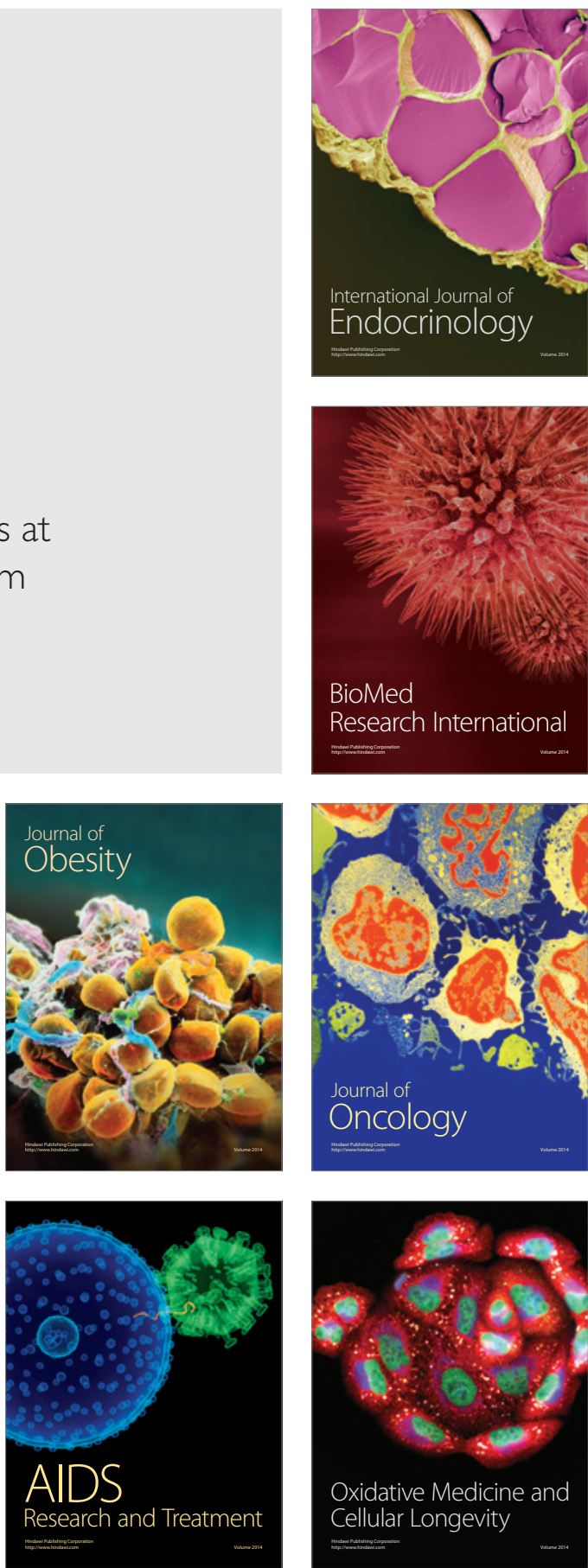